\section{Neues Wirkprinzip bringt Hoffnung für die Behandlung von metastasiertem Brustkrebs}

Die Angiogenese-Hemmung ist eine völlig neue Strategie in der Krebsbehandlung: Dem Tumor wird durch diese zielgerichtete Therapie (targeted therapy) die Möglichkeit genommen, neue Blutgefäße zu bilden - eine Voraussetzung, um sich mit Nährstoffen und Sauerstoff zu versorgen und so zu wachsen und Metastasen zu bilden. Nachdem sich das neue Wirkprinzip in der Darmkrebstherapie etabliert hat, können auch Brustkrebspatientinnen hoffen. Studien haben einen klinischen Nutzen der Angiogenese-Hemmung in der Behandlung von metastasiertem Brustkrebs nachgewiesen.

Laut Statistik ist in Deutschland bei Frauen Brustkrebs mit 55100 Neuerkrankungen pro Jahr die häufigste Krebsart. Während Brustkrebs im frühen Stadium sehr oft heilbar ist, sind die Prognosen für fortgeschrittenen Brustkrebs schlechter. Eine Wende in der Behandlung dieser Patienten zeichnet sich durch das neue Wirkprinzip der Angiogenese-Hemmung ab. Ein Hauptvertreter dieses Wirkprinzips ist Bevacizumab (Avastin ${ }^{\circledR}$ ), das als erster und bisher einziger Angiogenese-Hemmer für die First-line-Therapie von metastasiertem Darmkrebs in Kombination mit einem etablierten Chemotherapieregime zugelassen ist. Bei sehr guter Verträglichkeit verlängert es hier signifikant das Überleben um 5 Monate und das progressionsfreie Überleben auf 10,6 Monate [1].

\section{Angiogenese lässt den Tumor wachsen}

Wächst der Tumor über mikroskopische Grenzen hinaus, kann die Versorgung mit Sauerstoff und Nährstoffen durch Diffusion alleine nicht mehr aufrechterhalten werden. Für eine weitere Größenzunahme müssen neue Blutgefäße gebildet werden (Angiogenese). $\mathrm{Zu}$ den wichtigs- ten Angiogenese stimulierenden Faktoren gehört der vaskuläre endotheliale Wachstumsfaktor VEGF (vascular endothelial growth factor), der auch in der Embryogenese und bei der Wundheilung eine entscheidende Rolle spielt. Angiogenese fördernde und hemmende Faktoren halten sich im gesunden Organismus die Waage und neutralisieren sich in ihrer Wirkung (angiogene Balance), so dass nicht unkontrolliert neue Blutgefäße gebildet werden. Dieses Gleichgewicht kippt, wenn der Tumor Angiogenese stimulierende Faktoren freisetzt, wodurch neue Blutgefäße gebildet werden (angiogener Switch) [2]. Bei der tumorbedingten Angiogenese wird VEGF vom Tumor freigesetzt und erreicht auf Endothelzellen benachbarter Blutgefäße spezifische Rezeptoren, an die es binden kann. Durch das Andocken wird die Bildung neuer und das Wachstum vorhandener Blutgefäße im und zum Tumor angeregt. Dies ist die Voraussetzung dafür, dass der Tumor wachsen und Metastasen bilden kann.

\section{Angiogenese-Hemmung mit Bevacizumab}

Bevacizumab ist der einzige Antikörper, der freies VEGF im Serum bindet und neutralisiert. Da VEGF nicht mehr an die Rezeptoren andocken kann, wird die Angiogenese gehemmt. Miller [3] konnte in einer randomisierten PhaseIII-Studie die Angiogenese-Hemmung als Wirkprinzip für die First-line-Therapie des lokal rezidivierten oder metastasierten Mammakarzinoms nachweisen. Eingeschlossen waren von Dezember 2001 bis Mai 2004722 Patientinnen, die auf zwei Studienarme verteilt wurden. Der erste Studienarm erhielt Paclitaxel $90 \mathrm{mg} / \mathrm{m}^{2}$ an den Tagen 1,8 und 15 alle 4 Wochen, der zweite Studienarm erhielt zusätzlich $10 \mathrm{mg} / \mathrm{kg}$ Bevacizumab alle 14 Tage. Primärer Endpunkt war das progressionsfreie Überleben, zusätzlich wurde die Ansprechrate untersucht. Die Ergebnisse der Zulassungsstudie waren mehr als viel versprechend: Durch die zusätzliche Gabe von Bevacizumab wurde das progressionsfreie Überleben von 6,7 auf 13,3 Monate $(\mathrm{p}<0,0001)$ und die Ansprechrate von 16,4 auf $36,2 \%$ statistisch signifikant mehr als verdoppelt $(\mathrm{p}<0,0001)$. Zahlen zum Gesamtüberleben liegen noch nicht abschließend vor.

\section{Zulassung für 2007 erwartet}

Auf Basis dieser Studie wurde im Juli 2006 in Europa ein Antrag auf Zulassung für Bevacizumab zur First-line-Therapie des metastasierten Brustkrebs eingereicht. Die Zulassung in Deutschland wird im ersten Quartal 2007 erwartet.

\section{Referenzen}

1 Hurwitz H, et al:Bevacizumab plus Irinotecan, Fluorouracil and Leucovorin for Metastatic Colorectal Cancer, N Engl J Med 2004;350:2335-2342.

2 Bergers G, Benjamin LE: Tumorigenesis and the angiogenic switch Nat Rev 2003;3.

3 Miller KD, et al: Breast Cancer Res Treat 2005;94:S6 (abstr 3, update, data on file).

Weitere Informationen bei

Roche Pharma AG

Öffentlichkeitsarbeit Pharma

Herrn Dr. H.-U. Jelitto

79630 Grenzach-Wyhlen

Tel. +49 7624 14-2400, Fax -3366

\title{
PharmaTicker+++ PharmaTicker+++ PharmaTicker+++ PharmaTicker+++
}

Fresenius Biotech GmbH. Bei der Behandlung von Krebspatienten mit symptomatischem malignen Aszites erwies sich der trifunktionale Antikörper catumaxomab als wirksam und verträglich. Dies zeigen jetzt erstmals vorgestellte Phase-II/III-Daten mit 129 Patientinnen mit Ovarialkarzinom, die einer therapeutischen Punktion der Bauchhöhle bedurften.

Weitere Informationen be

Fresenius Biotech GmbH

Konzern-Kommunikation

Dr. Bernd Ebeling

Tel. +49 6172 608-2378, Fax -2294

pr-fre@fresenius.de
Wyeth Pharma GmbH. Für hervorragende Leistungen im Bereich der Forschung und Entwicklung wurde Wyeth Pharmaceuticals mit dem «Management Team of the Year» Scrip Award ausgezeichnet. Eine Jury wählte das Wyeth Forschungs- und Entwicklungsteam aus einer Vielzahl Arzneimittelhersteller aus. Die Zeitschrift Scrip World Pharmaceutical News zählt zu den wichtigsten Veröffentlichungen in der pharmazeutischen Industrie.

Wyeth Pharma GmbH

Pressestelle, Anika Wichert

Tel. +49 251 204-2030, Fax -2033

WicherA@wyeth.com
Ortho Biotech Division of Janssen-Cilag GmbH. Der Proteasom-Inhibitor Velcade ${ }^{\circledR}$ (Bortezomib) hat im Dezember 2006 die FDA-Zulassung für die Rezidivtherapie des Mantelzell-Lymphoms erhalten. Damit darf der Wirkstoff nun in den USA sowohl bei Patienten mit diesem aggressiven Subtyp des Non-Hodgkin-Lymphoms als auch bei Erkrankten mit multiplem Myelom nach lediglich einer Vorbehandlung eingesetzt werden.

Weitere Informationen be

Janssen-Cilag GmbH

Kerstin Aschoff

Tel. +49 2137 955-412

kaschoff@jacde.jnj.com 


\section{Neue Daten zur Therapie von Brustkrebspatientinnen mit Femara $^{\circledR}$}

Beim San Antonio Breast Cancer Symposium im Dezember 2006 standen wieder neue Daten zur Behandlung von postmenopausalen Frauen mit Östrogenrezeptor(ER)-positivem Brustkrebs im Mittelpunkt.

In einer Studie mit 12 postmenopausalen Frauen mit ER-positivem, primärem Mammakarzinom, konnten Jürgen Geisler et al., Bergen, Norwegen, bestätigen, dass die Östrogenspiegel im Plasma und im Tumorgewebe unter einer Behandlung mit Femara ${ }^{\circledR}$ (Letrozol) stärker absinken als unter Anastrozol [1]. Welche Konsequenzen das für Wirkungsspektrum und Verträglichkeit der beiden Substanzen in der klinischen Anwendung habe, müsse in weiteren Studien geprüft werden.

Einen direkten Vergleich von Letrozol und Anastrozol im Hinblick auf Lebensqualitätsparameter, Verträglichkeit und Morbidität führten J. Michael Dixon et al. an 185 postmenopausalen Frauen mit invasivem, ER-positivem Mammakarzinom durch [2]. Die Patientinnen erhielten randomisiert im Crossover-Design zuerst 12 Wochen lang Letrozol $2,5 \mathrm{mg} / \mathrm{d}$, gefolgt von 12 Wochen Anastrozol $1 \mathrm{mg} / \mathrm{d}$ bzw. vice versa. Auch in dieser Studie waren die Estradiolwerte nach Letrozol-Behandlung niedriger als nach Anastrozol. Jeweils etwa ein Drittel aller Patientinnen gaben an, Letrozol, Anastrozol oder keinem der beiden Medikamente den Vorzug zu geben. Spektrum und Häufigkeit der Nebenwirkungen der beiden Aromatasehemmer waren vergleichbar. 19 Patientinnen brachen ihre Teilnahme an der Studie ab, davon 4 noch bevor sie die erste Studienmedikation erhalten hatten. 8 Patientinnen der Letrozolgruppe und 2 Patientinnen der Anastrozolgruppe brachen wegen $\mathrm{Ne}$ benwirkungen ab.
In einer aktuellen vergleichenden Analyse der beiden großen, kontrollierten Studien ATAC und BIG 1-98 unter Berücksichtigung unterschiedlicher Endpunkt-Definitionen kommen L.A. Vakaet et al., Brüssel, zu dem Ergebnis, dass Risikodifferenzen und relative Risikoreduktionen bei Letrozol (BIG 1-98) und Anastrozol (ATAC) für die untersuchten Endpunkte vergleichbar sind. Die Autoren planen eine formale, auf individuellen Patientendaten basierende Metaanalyse unter Berücksichtigung spezifischer Patientensubgruppen. Eine verlässliche Antwort auf die Frage, ob Letrozol und Anastrozol vergleichbare Wirksamkeit und Verträglichkeit zeigen, verspricht die derzeit durchgeführte Studie FACE (Femara vs Anastrozole Clinical Evaluation). Erste Ergebnisse werden in San Antonio 2007 erwartet.

Östrogenrezeptor-Antagonist als Therapieoption bei fortgeschrittenem Mammakarzinom

In der adjuvanten Therapie Hormonrezeptor(HR)-positiver Mammakarzinome und bei fortgeschrittener Erkrankung auch in der Firstline-Therapie werden bei postmenopausalen Patientinnen immer häufiger Nichtsteroidale Aromataseinhibitoren (NSAI) der dritten Generation, das heißt Letrozol oder Anastrozol, eingesetzt. Patientinnen, die unter dieser Behandlung ein Rezidiv oder eine Progression ihrer Tumorerkrankung erleiden, scheinen entsprechend den Hinweisen aus Phase-II-Studien von einer Weiterbehandlung mit dem steroidalen Aromatasehemmer Exemestan oder mit dem Estrogenrezeptor-Antagonisten Fulvestrant zu profitieren. In EFECT, einer ersten klinischen Studie der Phase III, wurden nun diese beiden Substanzen bei Patientinnen mit Progress unter NSAI miteinander verglichen. Insgesamt 693 Frauen wurden für die Studie rekrutiert. Die mediane Zeit bis zur Progression betrug in beiden Behandlungsarmen 3,7 Monate. Die Zeit bis zum Pro- gress, die objektive Responserate, und der Anteil der Patienten, die durch die Therapie einen klinischen Nutzen erfuhren, waren in beiden Behandlungsarmen praktisch gleich. Lediglich in der Dauer des Ansprechens ergab sich für Fulvestrant gegenüber Exemestan ein Vorteil von fast 4 Monaten (13,5 vs 9,8 Monate), der jedoch keine statistische Signifikanz erreichte. William Gradishar aus Chicago, der die Ergebnisse der Studie in San Antonio vorstellte, wies zusammenfassend darauf hin, dass Fulvestrant sich bei dieser Patientinnengruppe als vergleichbar wirksam und verträglich erwies wie Exemestan [3]. Ein zentraler Kritikpunkt an der Studie ist, dass Patientinnen mit Human Epidermal Growth Factor Rezeptor 2 (HER2)-positivem Tumor nicht gezielt identifiziert und - entsprechend aktuellem Therapiestandard - mit dem monoklonalen anti-HER2-Antikörper Trastuzumab behandelt wurden.

\section{Referenzen}

1 Geisler J, et al: Letrozole tablets suppress tissue and plasma estradiol (E2), estrone and estrone sulfate levels more effectively than anastrozole in postmenopausal women with breast cancer. San Antonio Breast Cancer Symposium 2006; \#103.

2 Dixon JM, et al: Anastrozole and letrozole tablets in an investigation and comparison of quality of life, tolerability and morbidity (ALIQUOT). San Antonio Breast Cancer Symposium 2006; \#105.

3 Gradishar W, et al: Fulvestrant versus exemestane following prior non-steroidal aromatase inhibitor therapy: first results from EFECT, a randomized, phase III trial in postmenopausal women with advanced breast cancer. San Antonio Breast Cancer Symposium 2006; $\# 12$.

Weitere Informationen bei

Novartis Pharma GmbH

Dr. Irene Roth

Leitung Kommunikation Onkologie

Tel. +49 911273 12-643, Fax -841

irene.roth@novartis.com

\section{PharmaTicker+++ PharmaTicker+++ PharmaTicker+++ PharmaTicker+++}

Sanofi-Aventis Deutschland GmbH. Im Dezember 2006 wurden die Ergebnisse der zweiten Interimsanalyse der Phase-III-Brustkrebsstudie BCIRG-006 bekannt gegeben. Die Daten zur Wirksamkeit und Sicherheit nach median 3-jährigem Follow-up bestätigen, dass die Behandlung mit Taxotere ${ }^{\circledR}$-haltigen Regimes in Kombination mit Herceptin ${ }^{\circledR}$ (Trastuzumab) bei Frauen mit HER2-positivem Brustkrebs im Frühstadium das erkrankungsfreie Intervall signifikant verlängert.

Sanofi-Aventis Deutschland GmbH

Cristina lannazzo

Tel. +49 69 305-24417, Fax -18802

cristina.iannazzo@sanofi-aventis.com
AstraZeneca GmbH. Auf dem 29. San Antonio Breast Cancer Symposium im Dezember 2006 wurden neue Daten vorgestellt, die das positive Nutzen/Risiko-Profil von Anastrozol (Arimidex ${ }^{\circledR}$ ) untermauern. Eine weitere Auswertung der ATAC-Studie (Arimidex Tamoxifen alone or in Combination) zeigte, dass unter Anastrozol signifikant weniger thromboembolische Ereignisse auftraten als unter Tamoxifen.

Weitere Informationen bei

AstraZeneca $\mathrm{GmbH}$

Frau Dagmar Hinz

Tel. +49 4103 708-3760

dagmar.hinz@astrazeneca.com
Roche Pharma AG. Das Komitee für Produkte der Humanmedizin der Europäischen Union (Committee for Human Medicinal Products/ CHMP) hat im Januar die Zulassungsempfehlung für das oral applizierbare Krebsmedikament Erlotinib $\left(\operatorname{Tarceva}^{\circledR}\right)$ in der Therapie des metastasierten Pankreaskarzinoms ausgesprochen.

Weitere Informationen bei

Roche Pharma AG

Herrn Dr. H.-U. Jelitto

Tel. +49 7624 14-2400, Fax -3366 